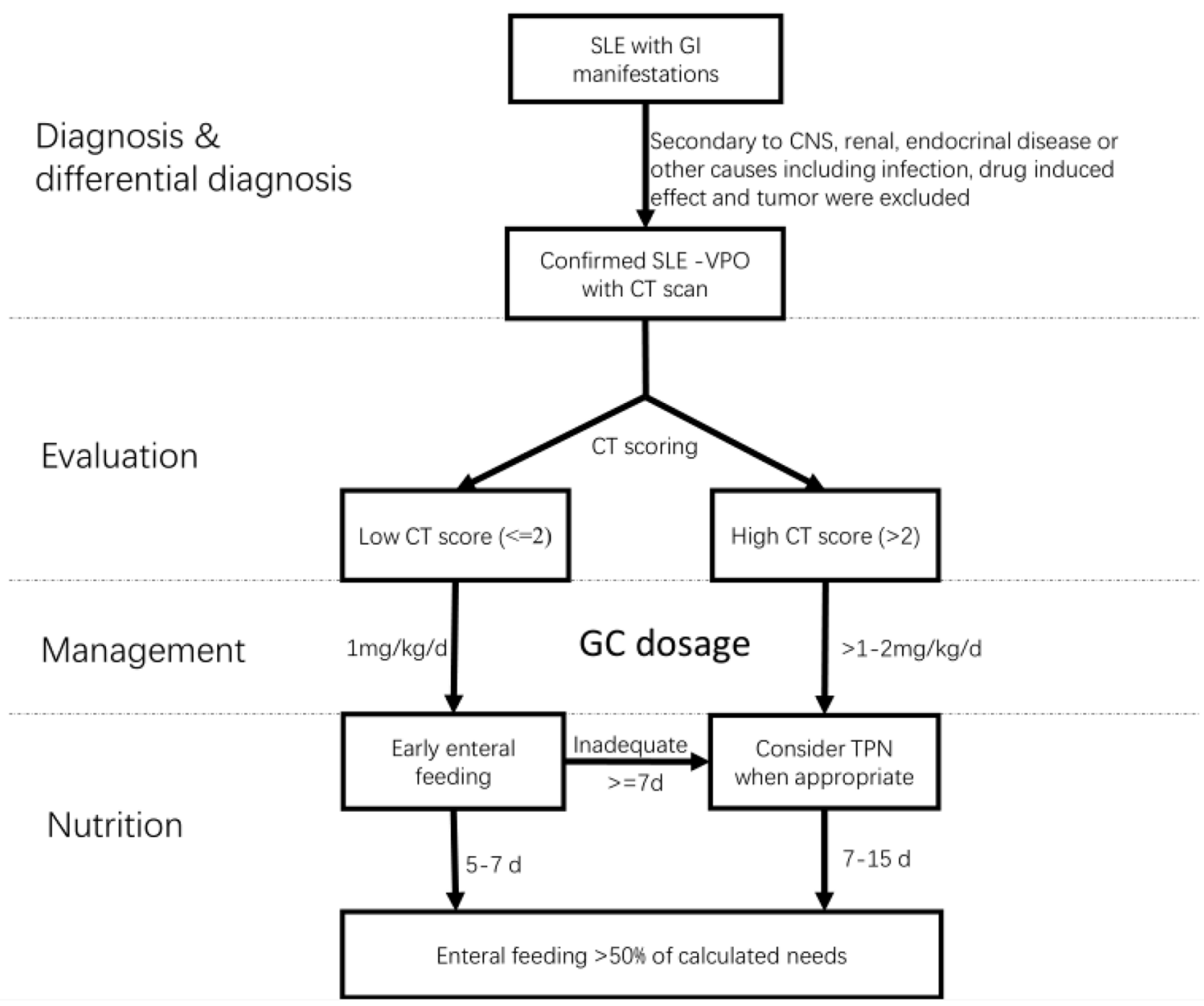

Abstract 28 Figure 4 A flow chart of SLE-VPO dietary recovery and TPN use based on CT scoring system

and $18.45(\mathrm{p}=0.0064)$ days, respectively. More TPN was prescribed in the high CT score group in both cohorts $(74.47 \%$ vs $51.16 \%, \mathrm{p}=0.0289$ in the derivation cohort; $80 \%$ vs $40.74 \%, \mathrm{p}=0.0089$ in the validation cohort). And the proportions of patients underwent TPN $\geq 7$ days were also much higher in the high CT score group in both cohorts $(\mathrm{p}<0.05)$.

Conclusions The terminology of VPO and a CT-based scoring system may facilitate more accurate assessment and individualized management for SLE patients with GI involvement. It also meant to be helpful in terms of future clinical trial design and in-depth mechanistic research for this unique complication of lupus.

Funding Source(s): This work was supported by the National key research and development program of China (2017YFC0909002), the National Key Research and Development Program of China(2016YFC0903900).

\section{SEROSITIS, HEMATOLOGIC INVOLVEMENT, AND STEROID DOSE ARE RISK FACTORS FOR SERIOUS INFECTIONS IN PATIENTS WITH SYSTEMIC LUPUS ERYTHEMATOSUS}

${ }^{1}$ Chang-Hee Suh*, ' $J u-Y a n g$ Jung, ${ }^{1}$ Dukyong Yoon, ${ }^{1}$ Hyoun-Ah Kim, ${ }^{2}$ Sang-Heon Lee. ${ }^{1}$ Ajou University School of Medicine; ${ }^{2}$ Konkuk University Medical Center

\subsection{6/lupus-2019-Ism.29}

Background Infection occurs frequently in patients with systemic lupus erythematosus (SLE) and has been a major cause of morbidity and mortality. However, no large-scale comprehensive studies have estimated the effect of clinical characteristics on serious infections in actual clinical practice yet. We investigated the influence of clinical characteristics on serious infections using electronic medical record data.

Methods We conducted a nested case-control study. Patients with SLE who developed serious infections needing hospitalization or intravenous antibiotic administration were matched to controls who did not. Odds ratios (ORs) and 95\% confidence intervals (CIs) for infection associated with the clinical characteristics were obtained using logistic regression analyses.

Results Among the total of 120 cases with infection, 93 $(77.5 \%)$ were bacterial infections with 40 (25\%) upper respiratory tract infections, $26(21.7 \%)$ pneumonia, 24 (20\%) sepsis, and $22(18.3 \%)$ urinary tract infections. The patients with serious infections had lower hemoglobin and C3 levels and less frequent hydroxychloroquine administration. In addition, they had more frequent nephritis, serositis, and hematologic involvement and took higher than the low dose of glucocorticoids (GCs; $>7.5 \mathrm{mg} / \mathrm{d}$ prednisolone-equivalent). The conditional logistic regression analysis with adjustment showed that serositis (OR, 2.76; 95\% CI, 1.33-5.74), hematologic involvement (OR, 2.53; 95\% CI, 1.32-4.87), and use of higher than the low dose of GCs (OR, 2.65; 95\% CI, 1.31-5.34) were related to serious infections in SLE

Conclusions Serositis, hematologic involvement, and use of higher than the low dose of GCs are risk factors for serious infections in patients with SLE. 
Funding Source(s): This research was supported by a grant from the Korea Health Technology R and D Project through the Korea Health Industry Development Institute, funded by the Ministry of Health and Welfare, Republic of Korea (grant number: HI16C0992).

\section{ASSOCIATION AND FUNCTIONAL STUDIES OF GENETIC POLYMORPHISMS OF MFG-E8 GENE IN SYSTEMIC LUPUS ERYTHEMATOSUS}

Wook-Young Baek*, Ju-Yang Jung, Chang-Hee Suh. Ajou University School of Medicine

\subsection{6/lupus-2019-Ism.30}

Background Systemic lupus erythematosus (SLE) is an autoimmune disease characterized by impaired clearance of apoptotic cells. Milk fat globule epidermal growth factor8 (MFGE8) is a protein connects between v3 integrin of phagocytic macrophage and phosphatidylserine of apoptotic cell surface. In this study, we determine whether genetic variation of MFG-E8 gene and serum protein concentration are associated with SLE.

Methods Single nucleotide polymorphisms (SNPs) were genotyped by three steps. At first, we used polymerase chain reaction in 20 patients with SLE and 20 normal controls (NC) for sequencing of a whole MFG-E8 gene in Korean population. Then we screened 12 selected SNPs in 55 SLE and 30 NC. Finally, we used Taq-man probe assay in 225 SLE and $230 \mathrm{NC}$ for genotyping of targeted 5 SNPs. Furthermore, serum MFG-E8 concentrations were analysed in SLE.

Results SLE patient's mean age was $35.7 \pm 7.8$ years and $92 \%$ were women, which is not different form NC. rs2271715's C allele and rs3743388's G allele were shown higher frequency in SLE than NC $(p=0.036, p=0.005$, respectively). As the linkage disequilibrium test, rs1878326 and rs1878327 were shown high linkage $\left(r^{2}=0.879\right)$. Three haplotypes were found by four SNPs (rs4945, rs1878327, rs2271715, and rs3743388); AACG, CGCG, and CGTC. The CGCG haplotype was significantly higher in patients with SLE compared with NC $(p=0.001$, odds ratio=2.31). rs4945 was associated with erythrocyte sedimentation rate and rs1878327 was associated with alopecia, C-reactive protein, complement 3, anti-double stranded deoxyribonucleic acid (anti-dsDNA) antibody, and systemic lupus erythematosus disease activity score. rs2271715 and rs3743388 were associated with renal disease, cumulated steroid dose, cyclophosphamide and mycophenolate mofetil usage and rs3743388 also was associated with anti-dsDNA antibody. Serum MFG-E8 concentration was shown significantly higher in SLE than NC $(2,030.3 \pm 1,292.3 \mathrm{pg} / \mathrm{mL}$ vs $1,433.0$ $\pm 946.3 \mathrm{pg} / \mathrm{mL}, \mathrm{p}=0.017)$.

Conclusions Our data suggest possibility that MFG-E8 rs2271715 and rs3743388 SNP can be involved in susceptibility of SLE. Also, these SNPs are associated with renal disease and disease activity in SLE. Furthermore, rs4945 and rs1878327 polymorphisms may be a marker of disease activity.

Funding Source(s): This research was supported by a grant of the Korea Health Technology $\mathrm{R}$ and $\mathrm{D}$ Project through the Korea Health Industry Development Institute (KHIDI), funded by the Ministry of Health and Welfare, Republic of Korea (grant number: HI16C0992).

\section{1 MINDFULNESS-BASED COGNITIVE THERAPY IN KOREAN PATIENTS WITH SYSTEMIC LUPUS ERYTHEMATOSUS}

${ }^{1}$ Hyoun-Ah Kim, 'Ju-Yang Jung, 'Wook-Young Baek, ${ }^{2}$ Sang-Heon Lee. 'Ajou University School of Medicine; ${ }^{2}$ Konkuk University Medical Center

\subsection{6/lupus-2019-|sm.31}

Background This study aims to evaluate the effectiveness of six-session stress reduction program based on mindfulness-based cognitive therapy (MBCT) among Korean patients with systemic lupus erythematosus (SLE) who experienced chronic stress.

Methods In total, 25 patients with SLE were enrolled. The MBCT program comprised two-hour sessions and homework for six weeks. The psychological data were collected through a questionnaire that included the Korean version of the Beck Depression Inventory-II (BDI-II), Beck Anxiety Inventory (BAI), Satisfaction with Life Scale (SWLS), and Perceived Stress Scale (PSS). We evaluated the effect of the MBCT on disease activity of SLE.

Results The mean scores on BDI-II, BAI, SWLS, and PSS before MBCT were 24.2 $\pm 10.6,19.1 \pm 9.7,14.7 \pm 6.5$, and 20.4 \pm 3.82 , respectively. Eighteen patients with SLE completed the MBCT program. After the MBCT, their BDI-II, BAI, and PSS improved; 17.4 $\pm 13.0(\mathrm{p}<0.01), 13.4 \pm 7.7(\mathrm{p}=0.04)$, and 17.9 $\pm 4.6(\mathrm{p}=0.04)$, respectively. However, SWLS score was not changed. There was no difference in disease activity between before and after the MBCT.

Conclusions This study showed preliminary evidence on the use of the MBCT in reducing the anxiety, depression, and stress of patients with SLE but not disease activity of SLE.

Funding Source(s): This research was supported by a grant from the Korea Health Technology R and D Project through the Korea Health Industry Development Institute (KHIDI), funded by the Ministry of Health and Welfare, Republic of Korea (Grant Number: HI16C0992).

\section{SAFETY, PHARMACOKINETICS AND PHARMACODYNAMICS OF BI 705564, A COVALENT INHIBITOR OF BRUTONS TYROSINE KINASE IN PHASE 1 CLINICAL TRIALS IN HEALTHY VOLUNTEERS}

${ }^{1}$ Tobias Litzenburger ${ }^{*},{ }^{1}$ Fabian Müller, ${ }^{2}$ Armin Schultz, ${ }^{3}$ Elliott Klein, ${ }^{3}$ Meera Ramanujam, ${ }^{3}$ Jing Wu, ${ }^{1}$ Sabrina Wiebe, ${ }^{1}$ Ewald Benediktus, ${ }^{3}$ Jianan Hui, ${ }^{3}$ Xiujiang Li, ${ }^{3}$ Mary Flack, ${ }^{4}$ Steven J Padula, ${ }^{5}$ Sudha Visvanathan, ${ }^{6}$ Jürgen Steffgen, ${ }^{1}$ Andreas Hünnemeyer. ${ }^{1}$ Boehringer Ingelheim Pharma GmbH and Co. KG, Biberach, Germany; ${ }^{2} \mathrm{CRS}$ Clinical Research Services Mannheim GmbH; ${ }^{3}$ Boehringer Ingelheim Pharmaceuticals, Inc., Ridgefield, CT, USA; ${ }^{4}$ Boehringer Ingelheim International, Ingelheim, Germany; ${ }^{5}$ Boehringer Ingelheim Pharmaceuticals, Inc., Ridgefield, CT, USA; ${ }^{6}$ Boehringer Ingelheim International, Biberach, Germany

\subsection{6/lupus-2019-Ism.32}

Background The small molecule BI 705564 is a highly selective, covalent and potent inhibitor (B cell CD69 activation IC50 $=2.2 \mathrm{nM}$ ) of the Brutons tyrosine kinase (BTK). BTK plays a critical role in the differentiation and function of $B$ cells and myeloid cell lineages and may play a major role in autoimmune diseases. Blocking the BTK pathways may be a promising new treatment of autoimmune diseases like SLE.

Methods BI 705564 has been studied in 43 male healthy volunteers (HV) in a single-blinded, partially randomized, placebo-controlled trial testing single rising doses from 10 to $160 \mathrm{mg}$. In a double-blinded, randomized, placebo-controlled 